\title{
LA IDEA AUSTRIACA DE “ESPÍRITU EMPRESARIAL” Y LAS INSTITUCIONES LABORALES EN LA ECONOMÍA GLOBAL
}

José Manuel Lasierra Esteban*

$\mathrm{E}$ 1 análisis económico evolucionó notablemente en el siglo pasado, de modo que hoy las fronteras entre escuelas son difusas y confluyen autores con inclinaciones antes divergentes, como ocurre con la escuela austriaca y la institucionalista. Este artículo analiza algunos aspectos de ambas escuelas con el fin de superar sus visiones parciales e incompletas y justificar una primera aproximación teórica que oriente análisis empíricos posteriores. El concepto de empresa reúne algunos de sus elementos esenciales que no se deben estudiar aisladamente si queremos entender la naturaleza de la empresa, su funcionamiento real y sus formas de organización.

Estas dos escuelas parten de supuestos diferentes, y distintos de los de la corriente neoclásica, aunque mantienen anclajes con la tradición ortodoxa. Uno de sus puntos de encuentro es la concepción de la empresa y del empresario, muy alejada de la visión neoclásica.

En el funcionamiento cotidiano de la empresa es normal la mejora continua y la búsqueda de información y de oportunidades, surgen costos de motivación y de coordinación entre directivos y empleados, y aparecen comportamientos oportunistas entre los agentes afectados por sus actividades. En suma, existen relaciones y comportamientos que la visión neoclásica no contempla, y que son difícilmente comprensibles si intentamos interpretarlos considerando por separado las ideas de ambas escuelas.

* Profesor titular de Economía Aplicada, Facultad de Ciencias Sociales y del Trabajo, Universidad de Zaragoza, Zaragoza, España, [jmlasie@unizar.es]. Fecha de recepción: 23-02-2016, fecha de modificación: 27-06-2016, fecha de aceptación: 20-10-2016. Sugerencia de citación: Lasierra E., J. M. "La idea austriaca de 'espíritu empresarial' y las instituciones laborales en la economía global", Revista de Economía Institucional 18, 35, 2016, pp. 79-100. DoI: http://dx.doi. org/10.18601/01245996.v18n35.05. 
La hipótesis de partida es que existen ciertas instituciones laborales que pueden reducir los costos de transacción (un aspecto de la economía institucional) en que incurren los agentes sin limitar la iniciativa empresarial (un aspecto básico de la escuela austriaca) y que incluso pueden estimularla, en economías abiertas con cambios técnicos y organizacionales. Para verificar la hipótesis se deben analizar los costos de transacción internos a la empresa y cómo son afectados por las instituciones.

En la primera sección se revisan la concepción austriaca de la empresa y las ideas fundamentales de algunos autores austriacos, como la de búsqueda y coordinación de la información, así como la visión dinámica del proceso de mercado y de "espíritu empresarial". En la segunda sección se revisa la visión institucionalista. En la tercera se analizan las complementariedades entre ambas escuelas. La información imperfecta y las relaciones de agencia, los costos de transacción, el tema de la equidad y el papel de ciertas acciones o valores colectivos son esenciales para entender el funcionamiento de la empresa. En la última sección se presentan algunas conclusiones y las consecuencias de política.

\section{LA ESCUELA AUSTRIACA}

La teoría neoclásica estudia en detalle las condiciones que facilitan la coordinación de las decisiones de los agentes mediante el sistema de precios, modela con elegancia el funcionamiento de los mercados competitivos y muestra el papel de los precios en la coordinación de las decisiones de numerosos agentes. E1 hecho de considerar el mercado como mecanismo único para asignar recursos ha limitado la comprensión de la naturaleza de la empresa. Para la economía neoclásica, la empresa es una ficción, una “caja negra”, dentro de la que nada ocurre ${ }^{1}$. Todos los fenómenos económicos se pueden reducir a proposiciones sobre las posiciones de equilibrio competitivo. Cada posición se examina tomando como referencia dicho equilibrio.

En el modelo neoclásico de equilibrio, general y parcial, está ausente el empresario. La empresa se representa como una función de producción y todo lo que ocurre en la empresa no aporta nada al análisis, no modifica ni altera la función de oferta ni tiene efectos sobre los resultados. E1 trabajo es un insumo homogéneo cuyo producto es

1 Ver Demsetz (1986), Fama (1984), Garrouste y Saussier (2005), Hamel y Prahalad (1997), Hayek (1945), Huerta (1997), Kirzner (1997), Lasierra (2001), March y Simon (1987), Nelson y Winter (1982), Santos (1997), Tirole (1990), Williamson (1989) y Williamson et al. (1975). 
predecible, no hay diferencias entre trabajo potencial y trabajo real y nada importa la organización interna de la empresa.

Lo que diferencia a la escuela austriaca moderna de la neoclásica es la visión de la competencia como un proceso de mercado en el que lo más importante es la búsqueda y el procesamiento de información, y el aprendizaje (Santos, 1997; Huerta, 1997). La información está dispersa y la actividad empresarial se desarrolla en un contexto de incertidumbre donde todos los agentes tienen oportunidades. Es pues una visión dinámica que se contrapone a la visión estática. Los austriacos comparten tres principios básicos.

1. $\mathrm{E} 1$ individualismo metodológico, que reduce las interacciones a relaciones de mercado (Fourie, 1994). Principio en el que coinciden con los neoclásicos y que parece alejarlos de los institucionalistas.

2. Su individualismo está impregnado de un subjetivismo que dificulta la medición y la comparación entre individuos, y lleva a rechazar el análisis cuantitativo (Ravier, 2012). El mercado es el medio para una asignación eficiente de los recursos donde los agentes encuentran la información dispersa y, si son buenos empresarios la saben interpretar $\mathrm{y}$ aprovechar. Cualquier agente que opere en el mercado puede ser buen empresario, pues el concepto se refiere a una actitud más que al estatus. En el límite, la empresa es un mercado en el que cada agente o trabajador vende su producto o servicio ${ }^{2}$, y paradójicamente la empresa no existe. Quizá por ello Kirzner no habla de empresa sino de "función empresarial".

3. El mercado nunca está en equilibrio sino en una incesante búsqueda de equilibrio que nunca se consigue, lo que llaman "proceso de mercado".

Uno de los mayores aportes de esta escuela es el desarrollo del concepto de empresario. Aunque la idea inicial surgió en la crítica de los primeros austriacos a la economía planificada ${ }^{3}$, muy pronto se reconoció el papel del empresario en la economía y en el sistema económico en general. A continuación revisamos brevemente los aportes de algunos autores al concepto de empresario.

Las primeras ideas sobre el empresario subjetivista se encuentran en Menger, para quien no cabe el error ni el conocimiento imperfecto en el ámbito económico. Nadie puede escapar a las leyes económi-

\footnotetext{
${ }^{2}$ La organización productiva se ha transformado: las empresas no solo compiten entre ellas en el mercado sino que dentro de ellas se han introducido mecanismos de competencia similares. La subcontratación y las unidades de negocio autónomas que compiten entre sí son nuevas formas de gestión que introducen los mercados dentro de la empresa (Cowen y Parker, 1998; Halal et al., 1993).

${ }^{3}$ Ver Menger (1871), Hayek (1945) y von Mises (1949).
} 
cas, que dadas las preferencias de los consumidores determinan los precios a partir de los cuales cada consumidor toma sus decisiones. Los aspectos subjetivos de la noción de Menger fueron ampliados por von Mises.

Para von Mises, la economía es una ciencia de la acción humana. La vida económica es la interacción entre agentes y cada acción es la unidad básica de análisis. Una persona actúa para sustituir un estado menos satisfactorio por otro más satisfactorio, de modo que subjetivismo e individualismo están unidos estrechamente. La acción humana tiene carácter intencional, su objetivo no es maximizar la eficiencia asignativa sino remover los obstáculos a la felicidad humana. En este punto tiene similitudes y diferencias con Robbins (1932). La acción humana de von Mises busca conseguir el fin propuesto, como prescribe Robbins, pero se diferencia en que al ser subjetivista configura la estructura de fines y los medios para alcanzarlos.

Como el subjetivismo es esencial, el análisis de las decisiones supera el marco estrecho de la asignación de recursos a unos fines dados. Para von Mises, en la acción se elige el marco relevante de fines y medios, y esa elección refleja los sueños de los agentes, sus aspiraciones, expectativas y conocimientos. Esta visión subjetiva sustituye la asignación mecánica del agente de Robbins, estática por naturaleza, por la dinámica de la acción humana. Von Mises define la función empresarial como la acción humana que considera la incertidumbre: "empresario quiere decir un hombre que actúa de acuerdo con los cambios que tienen lugar en el mercado".

Para Robbins (1932), el problema económico es determinar la combinación de recursos que logra de modo eficiente un fin dado. Esta conducta economizadora no determina los fines. El agente de von Mises, en cambio, no solo busca conseguir unos objetivos una vez dados los fines y medios, sino que está alerta para identificar los fines y elegir los recursos. No está constreñido a un mundo en el que todas las cosas son o tienen que ser conocidas, o lo serán pronto en un proceso sistemático de búsqueda. Esta ampliación del ámbito de acción permite descubrir oportunidades hasta entonces desconocidas, $y$ es la esencia de lo que los austriacos llaman función empresarial.

Con Schumpeter (1978 y 1996) el estudio del empresario y de la organización interna cobró un interés hasta entonces casi inexistente. Autores que en algún momento consideraron que el papel del empresario era distinto del trabajo o el capital, como Cantillon, Say o Walras, apenas le dedicaron un breve comentario. La visión de la empresa de Schumpeter integra varios elementos: el empresario 
individual y su función en la economía, la idea de individualidad, la iniciativa sujeta a intuición, la endogeneización de la tecnología y la organización como medio para ganar posición en el mercado, adelantarse a los demás y conseguir rentas extraordinarias, en un horizonte de desequilibrio permanente.

Su concepto de empresario no es unívoco; como señalan diversos autores, tiene dos significados ${ }^{4}$. $\mathrm{El}$ primero es de carácter personal, intuitivo, descubridor y heroico; esta dimensión individual enlaza con la de von Mises y su visión de la acción humana. Aunque se separa de ella cuando describe al empresario como una figura excepcional. Schumpeter no concuerda con la afirmación de que "todos somos empresarios", de que toda acción humana tiene un componente empresarial. El segundo significado se refiere al papel innovador y desequilibrador de las empresas, en particular de las grandes frente a las pequeñas; y en aquellas, del papel del empresario, cuya función sigue siendo excepcional pero ya no basada en cualidades personales sino en los recursos que puede dedicar a la innovación, mayores en las grandes empresas que en las pequeñas, en especial si puede obtener rentas de monopolio.

Schumpeter señala que las innovaciones económicas no surgen debido a nuevas necesidades espontáneas de los consumidores, que luego se difunden al aparato productivo. El productor inicia el cambio económico, y educa a los consumidores de ser necesario; les enseña a necesitar bienes que difieren en algún aspecto de los ya existentes. Esta idea es un antecedente de la orientación moderna de la empresa a la producción -después de un largo periodo de organización o gestión centrado en la demanda- que consiste en crear necesidades en vez de atender la demanda. Esta orientación enlaza con las ideas de austriacos modernos como Kirzner (1975 y 1992) o Loasby (1991). Autores que estudian las prácticas de gestión, como Hamel y Prahalad (1997) señalan algo parecido: "aprender a olvidar las viejas prácticas para incorporar más fácilmente las nuevas".

Si bien Schumpeter introdujo la figura del empresario y la dinámica en el análisis económico, los austriacos depuraron los aspectos del mercado y del papel del empresario en los que la tradición neoclásica del equilibrio mostraba graves deficiencias explicativas.

Hayek (1945) recalcó el individualismo en la tarea de aprovechar la información dispersa que aporta el mercado. La unión de la función empresarial de von Mises y del papel que Hayek atribuye al

${ }^{4}$ Ver Freeman et al. (1985), Kamien y Schwartz (1989) y Nelson y Winter (1982). 
mercado en la transmisión de información hace posible que Kirzner profundice el concepto de empresario propuesto por Schumpeter. Llevado al límite, el empresario del primer Schumpeter y el gestor de rutinas kirzneriano cumplen papeles complementarios en la empresa. El sistema económico se basa en los flujos de información. Y el problema económico es establecer una división apropiada del trabajo para procesar la información e invertir en la infraestructura correspondiente (Casson, 2005) 5 .

Kirzner $(1992,1997)$, como otros austriacos modernos, ve el mercado como un proceso sistemático de descubrimiento mutuo entre sus participantes. En ese proceso, en esa búsqueda de equilibrio nunca alcanzado, cumplen un papel fundamental el empresario de von Mises y el mercado de Hayek. Kirzner considera que hay muchas lagunas en el conocimiento, y que las fuerzas del mercado, puestas en marcha por la iniciativa empresarial, tienden a reducir la ignorancia. Así, el mercado proporciona conocimientos y facilita la coordinación. Aunque en las etapas del proceso de mercado se corrige la ignorancia inicial, este proceso no es una serie de actos deliberados de aprendizaje. El aprendizaje es la transformación de la ignorancia en conocimiento. Kirzner distingue el simple conocimiento, producto del aprendizaje continuo, que cualquiera puede conseguir dedicando recursos, del "descubrimiento", la esencia del proceso de mercado, aquella situación en la que "no se sabe lo que no se sabe", que exige del agente una continua disposición a descubrir.

E1 aprendizaje deliberado ocurre cuando se reconoce la falta de conocimiento, y se comparan sus ganancias con sus costos. $\mathrm{E} 1$ descubrimiento implica, en cambio, que no se es consciente de la ignorancia. Para Kirzner, el descubrimiento no es determinista ni mecánico por definición, de ahí que su proceso de mercado no se pueda modelar como un sistema de equilibrio, pues excluiría características esenciales del proceso de mercado. Lo esencial es la dimensión dinámica de la función empresarial, que es incompatible con situaciones siempre dadas, estáticas, de equilibrio, de recursos y objetivos dados que se maximizan à la Robbins. Kirzner recalca la disposición permanente a encontrar oportunidades de negocios y de beneficios y, por tanto, la función empresarial excluida por la teoría neoclásica de los precios.

\footnotetext{
${ }^{5}$ Algunos autores observan que no toda la información está dispersa y al alcance de todos. La investigación y el trabajo organizado -es decir, la empresa y la jerarquía- tienen aquí un papel importante (O’Neill, 2012).
} 


\section{LA EMPRESA COMO ORGANIZACIÓN UNA PERSPECTIVA INSTITUCIONALISTA}

Todo intento de explicar la organización empresarial debe considerar que la empresa ha cambiado enormemente desde su aparición como empresa industrial. Como señala Chandler (1977), la empresa moderna controla varias unidades que originan diversas transacciones coordinadas y vigiladas por empleados asalariados que sustituyen al mercado. Este autor, que analiza la aparición y el desarrollo de la empresa moderna, muestra que la empresa internalizó actividades que antes estaban dispersas entre diversas unidades y logró ventajas cuando creó una estructura gerencial capaz de dirigirla. La coordinación administrativa resultó más beneficiosa y eficiente que la coordinación a través del mercado.

En su historia de la dirección de la empresa, Pollard (1987) da ejemplo de formas de organización anteriores a la Revolución industrial que hoy parecen modernas o innovadoras, como las franquicias, el trabajo a domicilio y la subcontratación, pero que difieren en la capacidad técnica para controlar y coordinar las operaciones; en términos de Williamson, para considerar los costos de transacción, de coordinación y de motivación. La economía neoclásica no explica los cambios en la naturaleza de la empresa, por qué surgen las empresas ni cómo evolucionan, pues como ya se señaló, concibe la empresa como una función de producción, una "caja negra".

Coase (1937) sentó bases más firmes para entender la empresa, y dio origen a la visión contractualista que se desarrolló en los años setenta. Los primeros institucionalistas señalaron la importancia de las instituciones en el funcionamiento del sistema económico y examinaron los problemas distributivos, las relaciones de poder y la desigualdad económica. Insistieron menos en la eficiencia, como sí lo hace la nueva economía institucional desde una óptica institucional individualista (Toboso, 2001), un enfoque que han mantenido institucionalistas modernos como Solow (1992) y North (1984 y 1990).

\section{NUEVA ECONOMÍA INSTITUCIONAL: COSTOS DETRANSACCIÓN}

La economía de los costos de transacción (ECT) difiere de la economía neoclásica en su concepción del comportamiento de los agentes (Williamson, 1989). En primer lugar, la plena racionalidad neoclásica asegura la coherencia entre las premisas que guían las preferencias y la elección de los agentes, mientras que la racionalidad limitada de la ЕСT implica que así tengan objetivos definidos, los agentes pueden 
no tener información suficiente o tienen dificultades para procesarla. En segundo lugar, los agentes pueden actuar en forma oportunista o estratégica, es decir, incumplir los pactos. Además, los compromisos y contratos contienen indefiniciones e imprecisiones que intentan aprovechar en su propio beneficio.

Para Milgrom y Roberts (1992), el concepto transacción tiene dos sentidos: el de intercambio de bienes, servicios o dinero, y el de la menor unidad de la actividad económica, que no se puede subdividir para que la realicen agentes distintos. En una economía basada en la especialización y el intercambio, la actividad económica es resultado de una cadena de acciones elementales. La ест las llama transacciones, y tienen unos costos que la economía neoclásica no considera debido a sus supuestos: plena racionalidad, información completa, bienes estandarizados y perfecta sustitución de oferentes y demandantes. Milgrom y Roberts los dividen en costos de coordinación y costos de motivación. Los primeros están ligados a la obtención de información: sobre los precios relevantes, los oferentes y demandantes potenciales, su localización y las características de los bienes y servicios. Incluyen, además, los costos ocasionados por la falta de acople entre compradores y vendedores. Estos son de dos tipos: unos que son provocados por la información incompleta y la asimetría de información, y otros por las dificultades para cumplir lo pactado, el llamado compromiso imperfecto. Entre ellos, los de negociar y redactar los contratos, los costos en que se incurre para garantizar su cumplimiento o protegerse de los daños por incumplimiento.

Los costos de motivación aparecen cuando se relajan los supuestos del homo ceconomicus: la racionalidad ilimitada y la búsqueda del interés propio. Ya se comentó el primero. Williamson clasifica el segundo en tres tipos: búsqueda deshonesta, búsqueda honesta y obediencia. La segunda corresponde al supuesto neoclásico y la primera se denomina oportunismo. La economía neoclásica asocia la plena racionalidad a la búsqueda honesta mientras que la ECT, que tiene en cuenta la capacidad cognoscitiva real de los agentes, asocia la racionalidad limitada al oportunismo. Sin esos dos elementos se estrecha el margen para analizar la organización.

Desde el punto de vista contractual, la empresa es una estructura que sustituye al mercado en la función de dirigir y gobernar las relaciones económicas: los costos de transacción son determinantes en la elección de la manera de organizar las transacciones y las empresas.

Williamson identifica tres atributos de las transacciones: especificidad de los activos, incertidumbre y frecuencia de las transacciones. 
1. Especificidad. Los activos específicos son aquellos que tienen más valor en cierto entorno o relación que fuera de ella. Mientras que las transacciones neoclásicas son instantáneas, en mercados donde compradores y vendedores "sin rostro" intercambian productos estandarizados a precios de equilibrio, los intercambios asociados a inversiones específicas son transacciones que tienen rostro y no son inmediatas.

2.Incertidumbre. La incertidumbre está presente en todos los campos de la organización económica. El problema fundamental de la organización económica es cómo afrontar y manejar la incertidumbre (Koopmans, 1957). Existe una fuerte interrelación entre especificidad de los activos e incertidumbre. Cuanto mayor es la incertidumbre más se necesita un sistema de solución de disputas, pues los vacíos contractuales aumentan en número e importancia.

3. Frecuencia. Las transacciones poco comunes requieren estructuras de gestión especializadas que suelen ser más costosas. Una mayor frecuencia genera economías de comunicación debidas a la creación y el desarrollo de un lenguaje especializado a medida que se van acumulando experiencias. En las transacciones se pueden obtener ahorros por la relación que se establece entre proveedor y comprador a medida que los contratos se adaptan a las circunstancias y se llega a acuerdos.

Hasta ahora solo hemos mencionado la rama de la Ест que se ocupa de organizar las transacciones de modo que faciliten la adaptación eficiente. La otra rama es la de la medición, que busca la correspondencia más estrecha entre acciones y recompensas. Alchian y Demsetz (1972) también abordan el problema de la medición. Conviene recordar aquí que los problemas de gobierno o gestión y los de medición desaparecen si se adoptan los supuestos neoclásicos.

La concepción de las transacciones de la ECT permite analizar con más realismo la actividad económica que el modelo neoclásico. Pues en vez de considerar la empresa como una "caja negra", que utiliza insumos homogéneos para elaborar productos estandarizados, la concibe como una organización compleja, que adopta distintas estructuras de gestión y organiza de diversas maneras la división del trabajo y el intercambio mercantil.

\section{HACIA UNA VISIÓN INTEGRADORA.}

\section{INSTITUCIONES LABORALES Y ESPÍRITU EMPRESARIAL}

La empresa, o función empresarial, condensa los elementos de la escuela austriaca que destacan la adecuación y la adaptación permanente a las iniciativas de los competidores, en la búsqueda continua de nuevas 
oportunidades. Incluye, además, la noción de "ubicuidad empresarial" de von Mises, de empresarios que aprovechan la información dispersa de Hayek, la cual es indispensable para competir.

La empresa organiza las acciones individuales y reúne y procesa la información dispersa empleando diversos mecanismos de coordinación y de motivación, que dan lugar a estructuras que suelen diferir del modelo taylorista-fordista. Estos mecanismos funcionan de distintas maneras con costos distintos y producen resultados distintos. En las transacciones influyen las instituciones, y pueden ser más o menos costosas según sea el marco institucional, el cual proporciona criterios para diseñar esquemas organizativos.

$\mathrm{E}$ l individualismo metodológico de la escuela austriaca no puede explicar las características de la empresa actual sin los aportes de la ECT y de la visión institucionalista del mercado de trabajo. Dos puntos de vista contradictorios pero complementarios ${ }^{6}$. La ECT aporta elementos para diseñar esquemas organizativos y explicar su variedad, los límites de las empresas y la aparición de nuevos tipos de organización. $\mathrm{E} 1$ análisis del funcionamiento interno y de las relaciones entre los miembros de la empresa, de los objetivos dispares y de los conflictos entre trabajadores y directivos, de los incentivos como medio para reducir la ineficiencia provocada por la disparidad de intereses, de los costos de la estructura organizativa y del grado de centralización de las decisiones ha ampliado el campo de estudio de la empresa y de los determinantes de su competitividad. No es posible ignorar estos temas y ver el espíritu empresarial como manifestación única de la iniciativa individual, de agentes más o menos activos y emprendedores que actúan en el vacío.

La gráfica 1 sugiere que la realidad supera a la teoría. Muestra diversas formas organizativas, desde la estructura jerárquica tradicional hasta las relaciones empresariales de mercado puro. La parte derecha resume la forma taylorista-fordista, donde las relaciones son jerárquicas, las actividades están internalizadas y el empleo es relativamente duradero. Las relaciones entre la empresa y los trabajadores son reguladas por las normas laborales, los sindicatos y la negociación colectiva. A medida que se desplaza a la izquierda, la relación de empleo es cada vez menos regulada por normas laborales, que son sustituidas por normas mercantiles, hasta llegar al extremo izquierdo, donde operan relaciones mercantiles puras: acuerdos de compraventa o mercados. Hace tiempo las estructuras organizativas eran empresas semejantes a mercados puros. Hoy existen diversas formas que, dentro

${ }^{6}$ Como muestran Dequech (2006), Langlois (1984 y 1994) y Rutherford (1994). 
de la empresa y en las relaciones entre empresas, mezclan multitud de acuerdos y contratos. ¿Cómo explicar esa diversidad? A partir de la unidad mínima, la transacción, y de sus costos. Aunque la descripción anterior es incompleta si no incluye el factor que impulsa los cambios hacia y entre las diversas formas de empresa: la función empresarial.

Gráfica 1

La organización de la producción: del mercado a la empresa

(Un viaje de ida y vuelta)

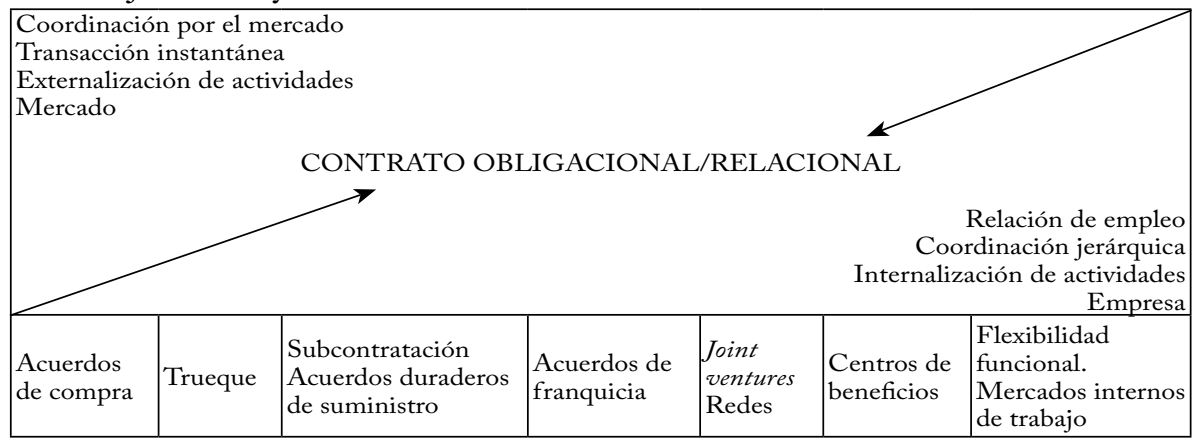

Fuente: elaboración propia.

Aunque no la racionalice, todo empresario tiene en mente la "función de producción de su empresa" y sabe que lo que sucede en la empresa es importante. Por ello busca y utiliza sistemas y tipos de organización adecuados para resolver los problemas que enfrenta la empresa. La gestión de alto rendimiento, es decir, las prácticas avanzadas de gestión de recursos humanos, contempla esos problemas y las diversas formas de organización, así como sus implicaciones, teniendo en cuenta que "todo se puede mejorar".

Todos los que participan en la tarea de explotar la información dispersa pueden encontrar oportunidades de mejora. La delimitación de las transacciones y la reducción de sus costos fomentan la iniciativa individual y estimulan el espíritu empresarial. A este respecto cabe mencionar algunas prácticas de gestión empresarial, como la mejora continua o el kaizen, que se pueden considerar como una aplicación del incrementalismo de Kirzner o Loasby, así como la gestión del trabajo y la gestión de calidad total.

Paradójicamente, la ECT aborda esos asuntos desde una óptica institucionalista que, combinada con el individualismo, ignora en cierta medida la acción colectiva de los agentes. Se puede afirmar que la teoría de los costos de transacción de Williamson explicaría mejor los problemas de racionalidad limitada y oportunismo si incorpora 
los aportes de la vieja economía institucional, que presta más atención a la redistribución y a la equidad, temas que también tratan autores neoinstitucionalistas como Solow, North y Simon. A continuación extraemos algunos elementos teóricos de ambas escuelas que ayudan a explicar la variedad de formas organizativas. De Williamson tomamos los riesgos del intercambio en el largo plazo y la idea de empresa como contrato incompleto.

Las relaciones de largo plazo no perduran cuando hay oportunidades fuera de la organización o el intercambio no produce ganancias. En el tiempo, las relaciones dependen de los costos y de inversiones específicas. Debido a la especificidad de los activos, las partes contratantes saben que luego habrá posibilidades de intercambio entre ellas, que producirán ganancias y podrán aprovechar. Lo esencial es que se distribuyan adecuadamente, es decir, que el intercambio sea eficiente ex post, de modo que la inversión específica sea eficiente $e x$ ante (Thurow, 1988). Las partes también pueden entrar en colusión, es decir, hacer acuerdos que compensen favores, no que funcionen eficientemente (Tirole, 1990). No obstante, la colusión puede reducir los costos de transacción porque limita el oportunismo en las relaciones de largo plazo. Debido a que la ruptura de estas relaciones puede ser rentable, el contrato debe encontrar un equilibrio entre flexibilidad y prevención del oportunismo.

En cuanto a la empresa como contrato incompleto, Tirole incorpora algunos elementos que no considera la visión neoclásica: la incertidumbre y las contingencias imprevistas en la ejecución del contrato. Tirole aborda estos elementos considerando la empresa como una manera de resolver los problemas a que dan lugar. Para él, dichas contingencias, que generan costos de transacción, son de dos tipos: las que son totalmente impredecibles a la hora de contratar, y las que son predecibles, pero muy numerosas para consignarlas en el contrato. Debido a tales contingencias, la supervisión de los contratos puede ser muy costosa y el costo de hacerlos cumplir puede ser muy alto. Entre la opción de no contratar y los contratos completos hay otros mecanismos o formas organizativas que facilitan la contratación. Entre ellos, recurrir a terceros para resolver los conflictos, el arbitraje y otorgar a una de las partes el derecho a resolverlos, es decir, otorgarle autoridad $^{7}$. La solución de los problemas que provocan los contratos incompletos explica en parte la mercantilización de las relaciones de trabajo: la subcontratación y el empleo free-lance (Dessein, 2014).

\footnotetext{
7 Este planteamiento es similar al de Alchian y Demsetz (1972). La autoridad que otorga la propiedad, la cual hace posible la coordinación y la solución de disputas, es la esencia de la función empresarial.
} 
La alternativa tradicional a la contratación era integrar las actividades en la empresa, y aún se sigue usando porque hace posible utilizar la reputación (Kimbrough y Rustin, 2015). Pero el comportamiento honesto ante las contingencias parece estar sustituyendo los contratos y la integración, pues en las relaciones entre empresas se observa una gran falta de formalismo. La confianza - la reputación- ahorra los costos de redactar contratos completos o de otorgar autoridad (Tirole, 1990). En el ámbito laboral, los contratos implícitos o el empleo de por vida en las empresas japonesas constituyen relaciones basadas en la confianza. Simon (1991) destaca el papel de las normas sociales y de las instituciones en la aceptación o el rechazo de ciertos comportamientos. North (1984) atribuye un papel similar a la ideología.

Existen pues nuevos elementos que se han de tener en cuenta. En el funcionamiento cotidiano de la empresa hay fricciones, pérdidas de tiempo y costos ocasionados por el desarrollo normal de la producción. No basta minimizar los costos de producción neoclásicos, también se deben reducir los costos de coordinación. La ECT considera algunas limitaciones o imperfecciones del contrato de trabajo, como las que ocasionan la racionalidad limitada y la no siempre bien resuelta distribución de los beneficios, que generan problemas de incentivos, de medida y valoración de los resultados, y comportamientos estratégicos. Es aquí donde la negociación colectiva, las normas laborales y ciertas prácticas o costumbres laborales pueden limitar el comportamiento oportunista, atenuar el problema distributivo, establecer relaciones de poder más equitativas en la empresa y reducir las asimetrías de información. Es decir, reducir los costos de transacción y contribuir a crear organizaciones más eficientes ${ }^{8}$.

A continuación se sintetizan los planteamientos de las escuelas austriaca e institucionalista sobre el papel de los mercados dentro de las organizaciones y las relaciones de poder internas.

1. El binomio tecnología-organización. A veces puede ser eficiente incurrir en mayores costos de producción. El sacrificio de economías de escala, si introduce la competencia dentro de la organización, puede producir mejores resultados finales porque la labor de los mercados es superior a la de cualquier organización. Por ello la gestión empresarial busca introducir mecanismos de mercado dentro de la empresa (Kleinknecht, 1998).

2. Las instituciones eficientes minimizan los costos de transacción. Esto no siempre es así, pues no se pueden ignorar las relaciones de poder que existen en las organizaciones. Los mismos supuestos de la

${ }^{8}$ Para más detalles, ver Lasierra (2001) y Auer et al. (2005). 
ECT, como el oportunismo, implican que los costos se trasladan a quien tiene menos poder". Eso también sucede debido al "efecto riqueza", es decir al hecho de que quien tiene poder lo usa en su provecho y puede generar resultados menos eficientes. Sayer y Walker (1994) señalan que en la obra de Williamson se excluye el poder. El hecho de que el desplazamiento del poder hacia la empresa traslade algunos costos a los empleados y genere un resultado económico global menor es un argumento contra la desregulación laboral ${ }^{10}$.

3. Incrementalismo y radicalismo. La visión incremental de la acción empresarial innovadora de Kirzner y Loasby difiere de la visión radical de Schumpeter, en cuanto que para los primeros hay un mejoramiento continuo y los empresarios suelen hacer mejoras no espectaculares (Volberda, 1998), aunque hacen una abstracción discutible: el empresario requiere la cooperación de otras personas, en particular de sus empleados, para aprovechar las oportunidades de mejora continua (Casson, 2005). Esto significa que la economía austriaca todavía ignora lo que ocurre dentro de la empresa, a diferencia de la ест. Hay una diferencia adicional en cuanto a la influencia de las instituciones formales e informales en las relaciones humanas y, por tanto en las relaciones laborales. La idea de mejora continua de la gestión moderna inspira las tesis de los modernos austriacos. E1 incrementalismo introduce dinamismo en las relaciones entre agentes, algo que falta en la visión neoclásica y que es la esencia de la escuela austriaca, a pesar de las críticas de la Ест. Grimshaw y Rubery (1998) critican los modelos de Williamson porque son estáticos cuando compara modos de organización eficientes. No obstante, esos modelos solo son ejemplos que ilustran su análisis. La crítica a la ECT porque hace comparaciones estáticas semejantes a las de la economía neoclásica no es válida puesto que, dados los supuestos de la ECT, su enfoque es dinámico. El problema es cómo enlazar la dinámica y la estática, es decir, qué sacrificar de la dinámica cuando se comparan organizaciones en un mismo momento (McGuinness, 1996). El empresario no está ausente en este análisis sino que lo hace posible, en contra de lo que dice Kirzner sobre las tesis de Williamson.

4. Simon señala que el intento de los institucionalistas de explicar el comportamiento organizativo en términos de agencia, información

\footnotetext{
${ }^{9}$ Milgrom y Roberts (1992) formulan el problema así: “¿por qué los empleadores deben minimizar los costos de transacción al diseñar sus sistemas de empleo, remuneración, promoción, supervisión y vigilancia, y no solo los costos que ellos mismos deben soportar?”.

${ }^{10}$ Como sucede con la desregulación laboral en España y en general en Europa, que rompió el equilibrio de poder entre empresas y sindicatos, y llevó al deterioro de los salarios y de las condiciones de trabajo.
} 
asimétrica, costos de transacción, oportunismo y otros conceptos neoclásicos ignora formas organizativas clave, como la autoridad, la identificación y la coordinación. Argumenta que la autoridad no se usa exclusiva ni principalmente para ordenar ciertas acciones. Muy a menudo las órdenes toman la forma de un resultado que se busca alcanzar, un principio que se debe aplicar o un objetivo que condiciona acciones sucesivas. Trabajar bien no es responder a las órdenes, sino tomar iniciativas para conseguir los objetivos de la organización. ¿Qué se requiere para ello, para que se trabaje en interés de la organización? Una respuesta simple es establecer un sistema apropiado para recompensar la contribución de cada cual. Una respuesta más compleja exige valorar el papel de las instituciones en la reducción del conflicto y del oportunismo, y en el estímulo de la cooperación (Kimbrough y Rubin, 2015) ${ }^{11}$.

Esos planteamientos tienen implicaciones prácticas para la empresa, la gestión de recursos humanos y las organizaciones. Los atributos de las transacciones de Williamson y las ideas de Simon sobre la autoridad, la identificación y la coordinación ayudan a afrontar con rigor el diseño de una organización económica eficiente.

Los fundamentos sobre los que se podría asentar tal organización se encuentran en Hayek y su noción de conocimiento disperso, que hace necesario establecer formas organizativas que lo aprovechen. También en Kirzner, para quien el aspecto dinámico de la actividad económica, que implica la adaptación continua a los cambios, es resultado de la búsqueda de nuevas oportunidades y de la acción de los agentes en busca del máximo beneficio, para los cuales la cooperación rinde más beneficios que la confrontación. Y por último, en la dimensión relacional donde opera la ECT. Además de esta relación entre comportamientos y organizaciones existen elementos técnicos, como las nuevas tecnologías, que aunque no son determinantes influyen en la elección de la estructura organizativa.

Hoy se debe modificar el viejo esquema de la relación hombremáquina que permite alcanzar ciertos niveles de eficiencia, pues la producción es más interdependiente y sistémica. Las tareas, funciones, empleos y empresas forman parte de una división del trabajo más intensa y compleja, que es necesario coordinar. La integración o cen-

\footnotetext{
11 Simon (1991) sostiene que la dificultad para cuantificar la relación entre contribución y recompensa es inherente a la organización. Según él, la identificación con la empresa induce a los empleados a aceptar sus objetivos y la autoridad. El orgullo por el trabajo y la lealtad son actitudes frecuentes y no excepcionales. Argumenta, además, que la identificación con la empresa es un poderoso instrumento para facilitar la coordinación interna, reducir el oportunismo y aumentar la eficiencia.
} 
tralización de actividades, característica de la empresa del siglo pasado, destacaba la cooperación interna o la jerarquía. La tendencia actual a unidades más pequeñas, a la descentralización y externalización exige no menos sino más colaboración y coordinación en materia de información, negociación y resolución de conflictos. En este contexto las instituciones laborales pueden ser efectivas para reducir los costos de transacción (Czeglédi, 2006).

Las instituciones contribuyen a establecer las reglas del juego, es decir los medios para el juego limpio que evitan el oportunismo y promueven el entendimiento. De alguna manera delimitan la estructura de incentivos y oportunidades y disminuyen los costos de transacción. Así, para cada operación los jugadores no van a tener que negociar los términos del contrato, con el consiguiente incremento de costos (Campbell, 1994). El incrementalismo, la mejora continua de procedimientos y el aprovechamiento de los recursos de la empresa, solo puede existir y fomentarse en un entorno de armonía y bienestar laboral, difícil de conseguir si no se promueve activamente. La gestión de recursos humanos en la empresa es importante, pero un entorno laboral de confianza la favorece. Aquí es donde las instituciones laborales, normas legales, sindicatos y negociación colectiva pueden desempeñar una labor importante y aun fundamental (Vergeer y Kleinknecht, 2014). Hay dos ámbitos, el interior de la empresa, la apertura de esa "caja negra" neoclásica, y la regulación laboral a nivel supraempresarial.

Por otra parte, las instituciones, más que un resultado de la producción, un producto que responde a cuestiones de equidad, son insumos necesarios para la eficiencia de la organización productiva moderna (Kleinknecht et al., 2013). Desde este punto de vista, la equidad es un medio para la eficiencia y el espíritu empresarial. La importancia económica de algunas instituciones laborales como la negociación colectiva, la costumbre o las normas laborales, según Sengenberger (1994a y 1994b), no debe analizarse únicamente desde la óptica estrecha de la empresa ya que el impacto del beneficio no es automático y universal. Más bien las instituciones deben considerarse como ingredientes de un desarrollo económico y social que afectará al sistema económico en general y a la empresa y sus agentes de forma más particular. En este sentido, aunque las instituciones pueden restringir la acción de los agentes, como señaló North (1984), también pueden desarrollar una función informativa-cognitiva (Dequech, 2006; North, 1990). 


\section{RESUMEN Y CONCLUSIONES}

La búsqueda de organizaciones eficientes es una gran preocupación. La eficiencia se asocia a la buena asignación de los recursos. El agente que busca y encuentra nuevas oportunidades impulsa la mejora continua y aumenta la eficiencia de la empresa y de todo el sistema. Este agente es el empresario, el emprendedor, que no está solo; en la empresa confluyen individuos e intereses que se deben gestionar bien para aprovechar las oportunidades y el mismo espíritu empresarial. La economía neoclásica ignora estos temas, mientras que las escuelas austriaca e institucionalista los abordan en forma parcial e incompleta. Argumentamos que si se examinan los aportes de algunos autores de estas escuelas, en especial su visión de la empresa, se encuentran sugerencias para fomentar la eficiencia de las organizaciones.

$\mathrm{E} 1$ análisis de esos aportes nos ha permitido encontrar elementos complementarios respecto de las funciones que pueden cumplir instituciones laborales como la negociación colectiva y la reglamentación laboral o incentivos que reduzcan el comportamiento estratégico y oportunista. La complementariedad de ambas escuelas se observa en el ámbito interno de la empresa y en el ámbito macroeconómico.

La conclusión básica es que las instituciones laborales pueden estimular la innovación y el crecimiento sin desincentivar la iniciativa empresarial. Para ello, las instituciones laborales y prácticas de gestión avanzada de los recursos humanos, que abren la "caja negra" neoclásica de la empresa, pueden desempeñar un papel fundamental, proactivo y no limitador y restrictivo del desarrollo empresarial. Lo que hemos pretendido destacar es la complementariedad que tienen estos ámbitos, el externo a la empresa y el interno. Dicho de otra manera, las instituciones laborales pueden estimular estrategias de recursos humanos y desarrollo empresarial más eficientes y contribuir a estimular el espíritu empresarial.

Este trabajo debería extenderse a la verificación práctica y empírica, si fuese susceptible de análisis cuantitativo, de las funciones que cumplen estas instituciones para generar organizaciones económicas eficientes. Menciona diversos trabajos que analizan el papel de las instituciones laborales en el crecimiento económico y la innovación. Este es un camino que cabría profundizar para valorar el papel de las instituciones, por ejemplo en la duración y el crecimiento de las empresas. Mencionamos por último algunas preguntas relacionadas con la política.

1. Si efectivamente se dan las relaciones de complementariedad por la influencia de las instituciones laborales, se trataría de ver cómo son, qué ventajas e inconvenientes plantean, cómo se relacionan con 
la acción pública, como por ejemplo las políticas reguladoras del mercado de trabajo (Lasierra, 2016), para desarrollar organizaciones eficientes y estimular el espíritu empresarial.

2. Si la información está diseminada y es necesario recogerla y utilizarla, con el fin de aprovechar todas las oportunidades, surgen algunas preguntas: ¿los sistemas de gestión que introducen mecanismos de mercado en la empresa, una aplicación de las ideas de Hayek, son más eficientes que las organizaciones jerárquicas, intervenidas por diversas instituciones laborales? ¿Cómo son esos mecanismos de mercado?

3. $\mathrm{Si}$, por el contrario, la función empresarial es más de "discovery" o de corazonada schumpeteriana, a lo mejor demanda unas políticas de empresa que premien a los "campeones" en un contexto laboral completamente desregulado. ¿Se desarrollará mejor al espíritu empresarial en un mercado de trabajo desregulado como el de Estados Unidos que en uno más intervenido como el europeo continental, francés o español?

4. Desde la perspectiva neoclásica, los mercados neoclásicos demandan trabajo neoclásico. Un trabajo homogéneo, con características perfectamente definidas, transparente como el agua: incoloro, inodoro e insípido. ¿Qué se exige, en ese contexto, de las políticas públicas? ¿Este tipo de organización estimula más el espíritu empresarial?

Estas preguntas sugieren caminos para proseguir la reflexión sobre la confluencia de dos escuelas de pensamiento en otro tiempo tan dispares.

\section{REFERENCIAS BIBLIOGRÁFICAS}

1. Abraham, K. y R. Mckersie, eds. New developments in the labor market: Toward a new institutional paradigm, Cambridge, Mass., Miт Press, 1990.

2. Alchian, A. y H. Demsetz. "Production, information costs, and economic organization", American Economic Review 62, 5, 1972, pp. 777-795.

3. Auer, P.; J. Berg e I. Coulibaly. "¿E1 trabajo estable mejora la productividad?", Revista Internacional del Trabajo 124, 3, 2005, pp. 345-372.

4. Baumol, W. y B. Blackman. Mercados perfectos y virtud natural. La ética en los negocios y la mano invisible, Madrid, Celeste-Colegio de Economistas, 1993.

5. Campbell, D. "The rationale for multi-level labour standards", W. Sengenberger y D. Campbell, eds., Creating economic opportunities: The role of labour standards in industrial restructuring, Ginebra, ILO, 1994.

6. Casson, M. "Entrepreneurship and the theory of the firm", Journal of Economic Behavior E Organization 58, 2, 2005, pp. 327-348.

7. Chandler, A. D. La mano visible. La revolución en la dirección de la empresa norteamericana [1977], Madrid, Centro de Publicaciones del Ministerio de Trabajo y Seguridad Social, 1987. 
8. Coase, R. La naturaleza de la empresa [1937], Madrid, Ariel, 1994.

9. Cowen, T. y D. Parker, Los mercados en la empresa. Mecanismos de mercado aplicados a la gestión, Madrid, Círculo de Empresarios, 1998.

10. Czeglédi, P. "Economic growth and institutional coherence", New Perspectives on Political Economy 2, 2, 2006, pp. 1-25.

11. Demsetz, H. La competencia. Aspectos económicos, jurídicos y politicos, Madrid, Alianza, 1986.

12. Dequech, D. "The new institutional economics and the theory of behaviour under uncertainty", Journal of Economic Behavior $\xi^{\circ}$ Organization 59, 2006, pp. 109-131.

13. Dessein, W. "Incomplete contracts and firm boundaries: New directions", Journal of Law, Economics E Organization 30, suplemento 1, 2014, pp. 113-136.

14. Fama, E. "Problemas de agencia y teoría de la empresa", Información Comercial Española 611, 1984, pp. 301-326.

15. Fourie, F. C. "In the beginning there were markets?", C. Pitelis, ed., Transactions costs, markets and hierarchies, Oxford, Blackwell, 1994.

16. Freeman, Ch.; L. Soete y J. Clark. Desempleo e innovación tecnológica, Madrid, Ministerio de Trabajo y Seguridad Social, 1985.

17. Garrouste, P y S. Saussier. "Looking for a theory of the firm: Future challenges", Journal of Economic Behavior E Organization 58, 2, 2005, pp. 178-199.

18. Grimshaw, D. y J. Rubery. "Integrating the internal and external labour markets”, Cambridge Journal of Economics 22, 2, 1998, pp. 199-220.

19. Halal, W. et al. Internal markets: Bringing the power of free enterprise inside your organization, Nueva York, Wiley, 1993.

20. Hamel, G. y C. Prahalad. Compitiendo por el futuro, Madrid, Ariel, 1997.

21. Hayek, F. "The use of knowledge in society", American Economic Review 35, 4, 1945, pp. 519-530.

22. Huerta de Soto, J. "La escuela austríaca moderna frente a la neoclásica", Revista de Economía Aplicada 15, 5, 1997, pp. 113-133.

23. Jensen, M. y W. Meckling. “Teoría de la empresa: la gerencia; costos de agencia y estructura de la propiedad" [1976], L. Putterman, ed., La naturaleza económica de la empresa, Alianza, Madrid, 1994.

24. Kamien, M. y N. Schwartz. Estructura de mercado e innovación, Madrid, Alianza, 1989.

25. Kay, N. "Markets, false hierarquies and the evolution of the modern corporation", Journal of Economic Behavior $\mathcal{F}^{\circ}$ Organization 17, 3, 1992, pp. 315-333.

26. Kimbrough, E. O. y J. Rubin. "Sustaining group reputation", Journal of Law, Economics E Organization 31, 3, 2015, pp. 599-628.

27. Kirzner, I. Competencia y función empresarial, Madrid, Unión Editorial, 1975.

28. Kirzner, I. The meaning of process markets, Nueva York, Routledge, 1992.

29. Kirzner, I. "Entreprenurial discovery and the competitive market process: An Austrian approach", Journal of Economic Literature 35, 1, 1997, pp. 60-85.

30. Kleinknecht, A. "Is labour market flexibility harmful to innovation?", Cambridge Journal of Economics 22, 3, 1998, pp. 387-396. 
31. Kleinknecht, A. et al. "Labour market rigidities can be useful: A Schumpeterian view", S. Fadda y P. Tridico, dirs., Financial crisis, labour markets and institutions, Abingdon, Routledge, 2013, pp. 175-191.

32. Kochan, T. A. y P. Osterman. The mutual gains enterprise, Cambridge, Mass., Harvard Business School Press, 1994.

33. Koopmans, T. Tres ensayos sobre el estado de la ciencia económica y los conceptos de optimalidad y su utilización, Barcelona, Antoni Bosch, 1957.

34. Langlois, R. "Internal organization in a dynamic context: Some theoretical considerations”, M. Jussawalla y H. Ebenfield, eds., Communication and information economics: New perspectives, Ámsterdam, North-Holland, 1984, pp. 23-49.

35. Langlois, R. "Risk and uncertainty", P. Boettke, ed., The Elgar Companion to Austrian Economics, Aldesht, Edward Elgar, 1994, pp. 118-122.

36. Lasierra E., J. M. "Regulación/desregulación laboral: una reflexión teórica”, mimeo, 2016.

37. Lasierra E., J. M. Mercado de trabajo y estrategias empresariales, Madrid, CEs, 2001.

38. Loasby, B. Equilibrium and evolution: An exploration of connecting principles in economics, Manchester, Manchester University Press, 1991.

39. March, J. y H. Simon. Teoría de la organización, Barcelona, Ariel, 1987.

40. McGuinness, T. "Markets and managerial strategies", G. Thompson et al., Markets, hierarchies and networks. The coordination of social life, Londres, Sage, 1996.

41. Menger, C. Principios de economía politica [1871], Barcelona, Unión Editorial, 1996.

42. Milgrom, P. y J. Roberts. Economics of organization and management, Englewood Cliffs, NJ, Prentice-Hall, 1992.

43. Mises, L. von. Human action: A treatise on economics, Londres, William Hodge, 1949.

44. Nelson, R. y S. Winter. An evolutionary theory of economic change, Cambridge, Mass., Harvard University Press, 1982.

45. North, D. Estructura y cambio en la bistoria económica, Madrid, Alianza, 1984.

46. North, D. Institutions, institutional change and economic performance, Cambridge, Mass., Cambridge University Press, 1990.

47. O'Neill, J. "Austrian economics and the limits of market”, Cambridge Journal of Economics 36, 5, 2012, pp. 1073-1090.

48. Pollard, S. La génesis de la dirección de la empresa moderna: estudio sobre la revolución industrial en Gran Bretaña, Madrid, Ministerio de Trabajo y Seguridad Social, 1987.

49. Ravier, A. "El marco analítico subjetivista en la economía del tiempo y de la ignorancia”, Revista de Instituciones, Ideas y Mercados 56, 2012, pp. 131-158.

50. Robbins, L. Essay on the nature and significance of economic science [1932], Londres, McMillan, 2007.

51. Rutherford, M. Institutions in economics, Cambridge, Mass., Cambridge University Press, 1994.

52. Santos R., M. Los economistas y la empresa, Madrid, Alianza, 1997. 
53. Sayer, A. y R. Walker. La nueva economía social, Madrid, Ministerio de Trabajo y Seguridad Social, 1994.

54. Schumpeter, J. A. Teoría del desenvolvimiento económico, México DF, Fondo de Cultura Económica, 1978.

55. Schumpeter, J. A. Capitalismo, socialismo y democracia, Barcelona, Orbis, 1996.

56. Schumpeter, J. A. Business cycles, Nueva York, McGraw-Hill, 1939.

57. Sengenberger, W. "Labour standards: An institutional framework for restructuring and development", W. Sengenberger y D. Campbell, eds., Creating economic opportunities: The role of labour standards in industrial restructuring, Ginebra, ILO, 1994a.

58. Sengenberger, W. "Protection-participation-promotion: The systemic nature and effects of labour standards", W. Sengenberger y D. Campbell, eds., Creating economic opportunities: The role of labour standards in industrial restructuring, Ginebra, ILO, 1994b.

59. Sengenberger, W. y D. Campbell, eds. Creating economic opportunities: The role of labour standards in industrial restructuring, Ginebra, ILO, 1994.

60. Simon, H. "Organizations and Markets", Journal of Economic Perspectives 5, 2, 1991, pp. 25-44.

61. Solow, R. El mercado de trabajo como institución social, Madrid, Alianza, 1992.

62. Thurow, L. Corrientes peligrosas: el estado de la ciencia económica, México DF, Fondo de Cultura Económica, 1988.

63. Tirole, J. La teoría de la organización industrial, Barcelona, Ariel, 1990.

64. Toboso, F. "Institutional individualism and institutional change: The search for a middle way mode of explanation", Cambridge Journal of Economics 25, 6, 2001, pp. 765-783.

65. Vergeer, R. y A. Kleinknecht. “¿Cómo afecta la desreglamentación a la productividad laboral? Análisis con datos de panel de 20 países de la ocde (1960-2004)", Revista Internacional del Trabajo 133, 3, 2014, pp. 403-434.

66. Volberda, H. Building the flexible firm, Oxford, Oxford University Press, 1998.

67. Williamson, O. E. Las instituciones económicas del capitalismo [1985], México DF, Fondo de Cultura Económica, 1989.

68. Williamson, O. "Markets, hierarchies, and the modern corporation. An unfolding perspective", Journal of Economic Behaviour E Organization 17, 3, 1992, pp. 335-352.

69. Williamson, O. "The new institutional economics: Taking stock, looking ahead", Journal of Economic Literature 38, 3, 2000, pp. 595-613.

70. Williamson, O.; M. Wachter y J. Harris. "La relación de empleo: el análisis del intercambio idiosincrásico" [1975], L. Toharia, comp., El mercado de trabajo: Teorias y aplicaciones, Madrid, Alianza, 1983.

71. Williamson, O. y S. Winter. The nature of the firm: Origins, evolution and development, Oxford, Oxford University Press, 1991. 
